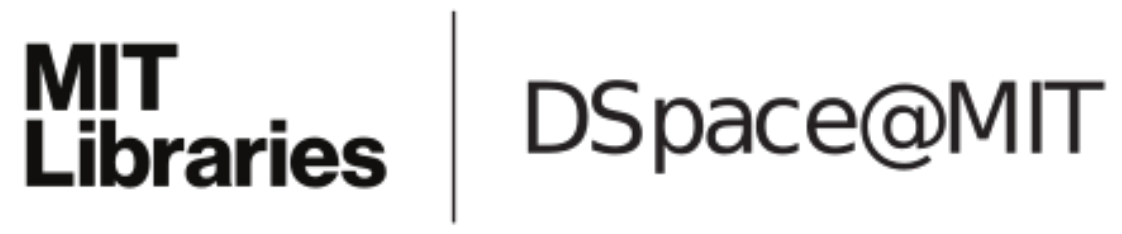

\author{
MIT Open Access Articles
}

Plastron Regeneration on Submerged Superhydrophobic Surfaces Using In Situ Gas Generation by Chemical Reaction

The MIT Faculty has made this article openly available. Please share how this access benefits you. Your story matters.

Citation: Panchanathan, Divya et al. "Plastron Regeneration on Submerged Superhydrophobic Surfaces Using In Situ Gas Generation by Chemical Reaction." ACS Applied Materials \& Interfaces 10, 39 (September 2018): 33684-33692 (c) 2018 American Chemical Society

As Published: http://dx.doi.org/10.1021/acsami.8b12471

Publisher: American Chemical Society (ACS)

Persistent URL: https://hdl.handle.net/1721.1/124202

Version: Author's final manuscript: final author's manuscript post peer review, without publisher's formatting or copy editing

Terms of Use: Article is made available in accordance with the publisher's policy and may be subject to US copyright law. Please refer to the publisher's site for terms of use. 


\title{
Plastron Regeneration on Submerged Superhydrophobic Surfaces using in-situ Gas Generation by Chemical Reaction
}

\author{
Divya Panchanathan, Anoop Rajappan, Kripa K. Varanasi, and Gareth H. \\ McKinley* \\ Department of Mechanical Engineering, Massachusetts Institute of Technology, Cambridge \\ E-mail: gareth@mit.edu
}

\begin{abstract}
Superhydrophobic surfaces submerged under water appear shiny due to total internal reflection of light from a thin layer of air (plastron) trapped in their surface texture. This entrapped air is advantageous for frictional drag reduction in various applications ranging from microfluidic channels to marine vessels. However, these aerophilic textures are prone to impregnation by water due to turbulent pressure fluctuations from external flows and dissolution of the trapped gas into the water. We demonstrate a novel chemical method to replenish the plastron in-situ by using the decomposition reaction of hydrogen peroxide on superhydrophobic surfaces prepared with a catalytic coating. We also provide a thermodynamic framework for designing superhydrophobic surfaces with optimal texture and chemistry for underwater plastron regeneration. We finally demonstrate the practical utility of this method by fabricating periodic microtextures on aluminum surfaces that incorporate a cheap catalyst, manganese dioxide. We perform drag reduction experiments under turbulent flow conditions in a Taylor-Couette cell (TC cell) which show that more than half of the drag increase ensuing from plastron
\end{abstract}


collapse can be recovered spontaneously by injection of dilute $\mathrm{H}_{2} \mathrm{O}_{2}$ into the TC cell.

Thus, we present a low-cost scalable method to enable in-situ plastron regeneration on large surfaces for marine applications.

\section{Introduction}

Superhydrophobic surfaces are well known for their properties of self-cleaning, ${ }^{1}$ fog collection, ${ }^{2}$ anti-icing, ${ }^{3}$ and anti-biofouling. ${ }^{4}$ The design of synthetic superhydrophobic surfaces is often inspired by naturally occurring water repellent surfaces such as lotus leaves and waterstrider feet. ${ }^{5}$ These naturally occurring surfaces have a hierarchical texture with microand nanoscale features, coupled with a hydrophobic chemical coating (Young's contact angle $\theta_{w}>90^{\circ}$ ) which collectively impart the property of super-repellency towards water (here super-repellency is taken to mean apparent contact angle $\theta_{w}^{*}>150^{\circ}$, and a roll-off angle $\left.<5-10^{\circ}\right) \cdot \cdot^{1,6,7}$ When a superhydrophobic surface is submerged underwater, a thin layer of air is trapped within its microtexture corresponding to the three phase configuration known as the Cassie state. ${ }^{8}$ Hence, these textures are also known as underwater superaerophilic surfaces. ${ }^{9,10}$ The air entrapped within the texture is termed a plastron in the biological community and this phenomena is observed in nature among many insects and plants which have evolved the ability to breathe underwater. ${ }^{11-13}$ In addition to preventing water from wetting the underlying substrate, the plastron acts as a barrier towards corrosion on metal surfaces ${ }^{14}$ and the surface air layer reduces the skin friction drag experienced by superhydrophobic surfaces underwater. ${ }^{15,16}$

Continued stability of the Cassie state is essential for exploiting the desirable properties of underwater superaerophilic surfaces. But the air in this three-phase state is easily displaced by water under pressure fluctuations, shear flow, and low dissolved gas concentrations ${ }^{17-21}$ causing them to transition into the Wenzel or fully-wetted state. ${ }^{22}$ In order for such a surface to recover its drag-reducing and anti-corrosion properties, it is necessary to induce a Wenzel-to-Cassie state transition on the textured surface while it remains submerged in the 
liquid phase. Wenzel-to-Cassie state transitions on textured surfaces have been achieved for sessile water droplets in air using electrowetting, ${ }^{23,24}$ boiling, ${ }^{25}$ modulation of the Laplace pressure, ${ }^{26}$ vibration, ${ }^{27}$ and volatile low surface tension liquids. ${ }^{28}$ However, these methods (except boiling) cannot easily be applied when the surface is submerged underwater because there is no source of air available in the surroundings to displace the water in the texture. Therefore in order to actively restore a plastron by replenishing the Cassie state, there is a clear need to implement a gas or vapor source underwater. Methods that have been developed to address this need can be broadly classified into three categories: (i) in-situ gas/vapor generation, (ii) gas injection, and (iii) increasing the concentration of dissolved gas in the contacting liquid.

The first approach uses in-situ generation of gas/vapor to replenish the gaseous/vapor phase that initially formed the lost plastron. These regenerative techniques include electrolysis, ${ }^{29,30}$ photoelectrocatalytic water splitting, ${ }^{31}$ and film boiling. ${ }^{32,33}$ Lee et al. demonstrated in-situ plastron recovery using electrolysis on $\mathrm{Pt} / \mathrm{Au}$ coated silicon pillars. ${ }^{29}$ Although this method has the advantage of being self-limiting, it is expensive to perform water-splitting using noble metals. On the other hand, film boiling has no material limitations but is energy intensive to perform on a large scale. ${ }^{34}$ The second method, gas injection, relies on providing a continuous supply of gas/air to the plastron. This can be achieved using a connected plastron (plastron connected to an air reservoir through a porous microstructure, ${ }^{35}$ a large bubble ${ }^{36}$ or a region of compressed gas ${ }^{37}$ ), or by injecting gas bubbles directly into the boundary layer of the flow. ${ }^{38}$ Connecting a plastron to an external reservoir does not require any expensive materials or energy input, but needs precise control of pressure in the reservoir to favor plastron spreading. Also, bubbles injected into the boundary layer do not adhere to the surface immediately due to the lubrication pressure exerted on the bubble by the draining liquid film. ${ }^{39}$ These methods are typically not self-limiting and are susceptible to the formation of large bubbles that reside on the texture instead of forming a thin plastron layer within the texture. ${ }^{38}$ These macroscopic bubbles increase the form drag by disrupting 
the boundary layer, even though the skin friction drag contribution is lower. ${ }^{40}$ The final approach is to saturate the water with dissolved air/gas to achieve plastron recovery by diffusion through the liquid and mass transfer at the interface. ${ }^{41,42}$ This technique requires the gas concentration to be controlled locally near the surface by increasing the temperature or decreasing the pressure of the water in situ.

In this work, we demonstrate a novel chemical method to replenish air loss from an established plastron by using catalytic decomposition of hydrogen peroxide. Hydrogen peroxide is an unstable compound which slowly decomposes into water and oxygen (Equation 1) even at room temperature and pressure.

$$
2 \mathrm{H}_{2} \mathrm{O}_{2} \stackrel{\text { catalyst/heat }}{\longrightarrow} 2 \mathrm{H}_{2} \mathrm{O}+\mathrm{O}_{2}
$$

This reaction is slow under room temperature conditions (with a decomposition halflife of $t_{1 / 2} \sim 3$ years $^{43}$ ) but can be accelerated by increasing the temperature or using a suitable catalyst. There are a range of possible catalysts including the enzyme catalase, ${ }^{44}$ platinum and its alloys, ${ }^{45}$ iodide $^{46}$ and metal oxides. ${ }^{47}$ This reaction has been previously recently exploited in studies of active matter which use gas generation to propel nanorods and microtubes underwater. ${ }^{48,49}$ In this work, we use the overpressure of the oxygen gas generated by the decomposition reaction to dewet the microtexture and drive a wetting state transition from the Wenzel state back to the Cassie state. Figure 1 depicts the stages of plastron failure-recovery cycle using the chemical gas generation concept. In the first stage, the superhydrophobic surface 'fails' or transitions from Cassie to Wenzel state upon impregnation by water e.g. due to large external pressure fluctuations from turbulent flow. At this point, dilute concentrations of hydrogen peroxide added to the bulk liquid can react with the catalyst deposited at the bottom of the wetted microtexture and generates gas which spreads through the textured surface laterally, until ultimately the plastron has been fully restored. Because the reaction only occurs where the catalyst is in direct contact with the $\mathrm{H}_{2} \mathrm{O}_{2}$ solution (i.e. in regions where the plastron has collapsed), it is self-limiting. 
Furthermore, it requires only dilute concentrations (3-4\%) of the reactant, hydrogen peroxide, to observe plastron recovery. It also does not require electrical power input and can be performed using cheaper catalysts than those typically used in electrolytic water-splitting. This cycle can be repeated multiple times until the reactant is depleted or the catalyst is degraded.

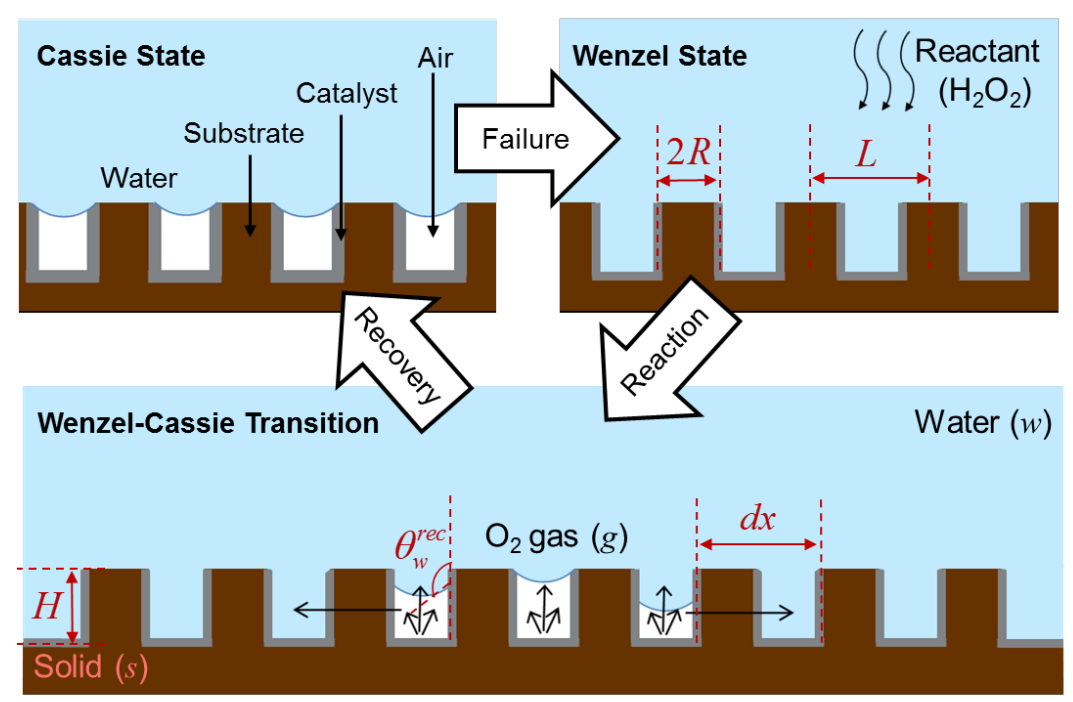

Figure 1: Schematic of an active superhydrophobic microtexture which generates gas ondemand upon addition of the reactant, hydrogen peroxide.

Plastron recovery occurs only when the conditions are optimal for lateral gas spreading. Lee et al. ${ }^{29}$ derived a criterion for plastron recovery by stipulating that the lateral breakthrough pressure must be lower than the vertical breakthrough pressure. Forsberg et al. ${ }^{37}$ obtained another condition on the premise that the lateral front of the plastron must touch the successive pillar before the vertical front reaches the top of the microtexture. Lee et al. ${ }^{29}$ and Verho et al. ${ }^{36}$ found that a superimposed nanotexture is a necessary condition for plastron recovery; by contrast, Forsberg et. al. obtained plastron recovery on pillared microtextures with smooth walls. One of the reasons for the inconsistency in these observations is that the corresponding theories were derived based on geometric principles and the inherent thermodynamic stability of the plastron in the texture was ignored. Jones et al. ${ }^{50}$ and Marmur et al. ${ }^{51}$ show that the stability of the Cassie state is the primary condition for 
underwater stability of the plastron. We explore the importance of this condition for the lateral spreading of the plastron in an underwater superhydrophobic texture.

We fabricate micropillars interspersed with platinum catalyst on silicon wafers, hydrophobize them with a Teflon layer, and demonstrate plastron recovery underwater when dilute concentrations of hydrogen peroxide are introduced. We propose a thermodynamic model based on energetics of gas hemiwicking underwater, and derive a criterion for plastron recovery. We explore this criterion using platinum-coated micropillar arrays of varying height and pitch. Finally, we also demonstrate practical plastron recovery during measurements of frictional drag on an inexpensive and scalable aluminum surface with manganese dioxide catalyst using a Taylor-Couette cell. ${ }^{52}$

\section{Thermodynamic States of Gas Bubbles on Aerophilic Tex-}

\section{tures}

Bubbles interact with textured solid surfaces in different ways depending on the roughness and chemistry of the surface. ${ }^{53}$ We classify the states of wetting of a gas bubble generated on a textured solid surface immersed underwater based on their thermodynamic stability. Comparison with the corresponding system in which a drop of liquid wets a textured solid surface in air enables the identification of four distinct regimes, and the stability of each of these gas bubble-in-water states depends on both the surface chemistry and roughness of the texture. Figure 2a shows a schematic illustrating the four regimes on a microtextured solid surface - bubble, blister, hemiwicking blister, and plastron. Bubble identifies the state in which the gas does not want to wet the solid surface and is pinned to the surface with minimal solid-air contact area. Blisters are bubbles that partially spread on the solid surface until they they establish an equilibrium pressure and apparent contact angle. They do not imbibe spontaneously into the texture. The Plastron is the state in which the gas is completely imbibed in the texture and only the top of the surface texture contacts the liquid phase. 
Finally, the intermediate state identified as Hemiwicking Blisters are blisters that spread on top of a microtexture imbibed with air i.e. plastron. The distinction between a blister and a hemiwicking blister is that the fluid phase preferentially imbibed in the texture surrounding the gas blister is different. We define this state using a nomenclature derived from the work of Bico et al. who considered corresponding states for liquid droplets wetting microtextured dry substrates in air. ${ }^{54}$ It can be seen from detailed examination of Figure 2a (i)-(iv) that the pillars are visible for the bubble and blister states while the pillars are hidden inside the gas layer for the hemiwicking blister and plastron states. When gas is produced in the texture through the decomposition of hydrogen peroxide, it may leave the surface through buoyancy effects as a macroscopic bubble, or spread through the microscopic texture in one of the other states depending on the texture chemistry and roughness, and the rate of generation compared to the rate of spreading.

In this work, we follow Quéré's hemiwicking model for hydrophilic textures ${ }^{54}$ to obtain an equivalent hemiwicking model for superhydrophobic textures underwater. In ideal plastron recovery, the gas would spread along the surface microtexture without displacing the water on top of the texture as depicted in Figure 1. This process involves the creation of new solidair and water-air interfaces while replacing the water-solid interfaces during de-wetting. The change in energy of the system when the gas front displaces water from a small area $l d x$ is given by the following equation.

$$
d E=\gamma_{w, g}\left(1-\phi_{s}\right) l d x+\gamma_{s, g}\left(r-\phi_{s}\right) l d x-\gamma_{s, w}\left(r-\phi_{s}\right) l d x
$$

where $\gamma_{w, g}, \gamma_{s, g}$ and $\gamma_{s, w}$ represent the respective interfacial energies between the three phases, $r$ and $\phi_{s}$ denote the Wenzel roughness and the projected solid fraction respectively, and $l$ is the length into the plane of Figure 1. The Wenzel roughness $r$ is defined as the total surface area per unit projected area of solid surface, and solid fraction $\phi_{s}$ is defined as the area wetted by the liquid in the Cassie state per unit projected surface area of solid. For example on a microtexture with circular pillars, $r$ and $\phi_{s}$ are calculated by using the following equations. 
a.
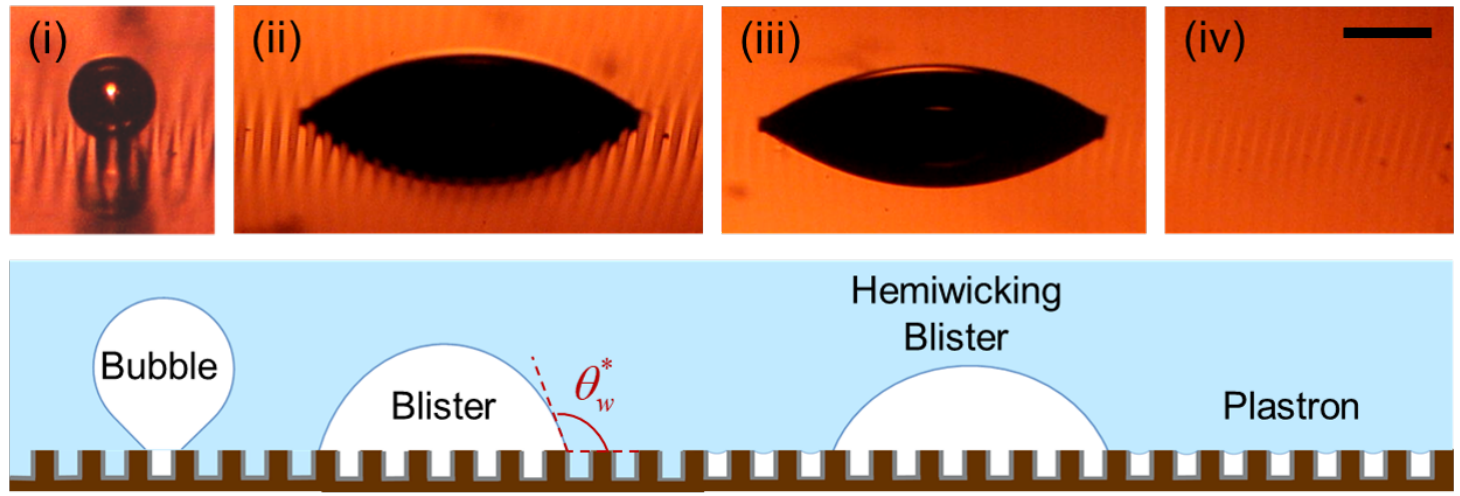

b.

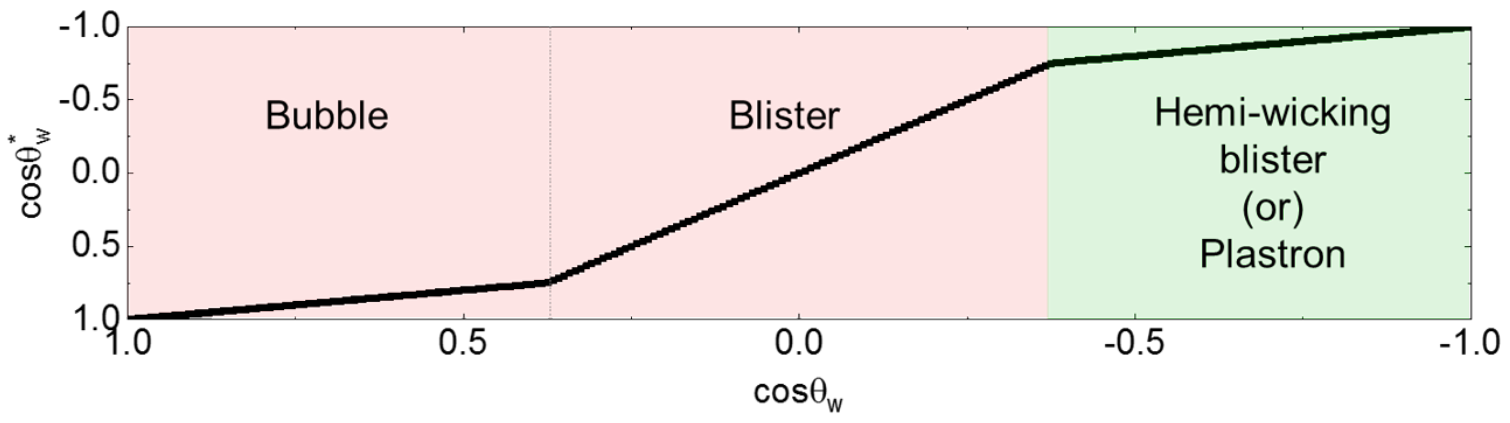

Figure 2: (a) Schematic of the thermodynamic states of gas bubbles spreading on textured surfaces. The stability of the states is dictated by both the microtexture and surface chemistry. The scale bar represents $0.5 \mathrm{~mm}$; (b) Plot of the apparent contact angle of water $\left(\theta_{w}^{*}\right)$ on a textured surface (Wenzel roughness $r=2$, solid fraction $\phi_{s}=0.4$ ) versus Young's contact angle of water on a flat surface $\left(\theta_{w}\right)$. The theoretically predicted regime of stable underwater gas wetting or aerophilicity is also indicated by the shading in the plot. Plastron recovery is favored only in the region shaded in green. 


$$
\begin{gathered}
r=1+\frac{2 \pi R H}{L^{2}} \\
\phi_{s}=\frac{\pi R^{2}}{L^{2}}
\end{gathered}
$$

The change in energy $d E$ of the system must be negative in order for the gas to preferentially wick into the texture. Hence by setting $d E<0$ and simplifying the expresion using the expression for Young's three phase contact angle equation, ${ }^{55}$ we obtain the thermodynamic condition for spontaneous plastron recovery.

$$
\cos \theta_{w}<-\frac{1-\phi_{s}}{r-\phi_{s}}
$$

Equation 5 implies that plastron recovery is only possible for textures with hydrophobic chemistries $\left(\theta_{w}>90^{\circ}\right)$ since the expression on the right is always negative. The favorable regimes of underwater gas wetting can be represented on the plot illustrating the relationship between the cosine of the apparent contact angle of a water droplet on a textured solid in air, $\cos \theta_{w}^{*}$ and the cosine of the Young's contact angle of a water droplet on a flat surface in air, $\cos \theta_{w}$ as shown in Figure $2 \mathrm{~b}$. Here it can be seen that plastron and hemi-wicking blisters are favorable in the textures which are Cassie-stable towards sessile water droplets in air, blisters are favorable in the textures which are Wenzel-stable towards sessile water droplets in air, and bubbles are favored in the textures which are hemiwicking towards sessile water droplets in air. Since gas displaces water in the texture during plastron spreading, it is most appropriate in Equation 5 to use the receding contact angle of a sessile water droplet on a smooth surface with the same chemistry. Rearranging Equation 5 using the receding water contact angle gives us the condition that needs to be satisfied for textural design parameters such as the solid area fraction $\phi_{s}$ and the solid roughness $r$. This also matches with the monostable Cassie state condition derived by Li et al. for the Wenzel-toCassie transition of liquid droplets on microtextures. ${ }^{26}$ For plastron recovery to be favorable 
over blister formation, we therefore require microtextures with roughness exceeding a critical value.

$$
r>1-\left(\frac{1}{\cos \theta_{w}^{r e c}}+1\right)\left(1-\phi_{s}\right) \quad \forall \theta_{w}^{r e c}>90^{\circ}
$$

\section{Results and Discussion}

In this work, we use two types of textured surfaces for testing plastron recovery - lithographically patterned silicon and laser-textured aluminum (See Figure 3). In order to systematically study the effect of surface roughness on plastron recovery, micro-pillar arrays with varying pillar heights and spacing were fabricated on silicon wafers using photolithography. Platinum, a well-known catalyst for the hydrogen peroxide decomposition reaction, was selectively deposited on the bottom and the sides of the micro-pillars using lift-off photolithography (see the experimental methods section for more details), to ensure that the plastron re-inflation process is self-limiting. The textures were then spin-coated with a 1 wt.\% Teflon solution and cured to render them hydrophobic.

Figure 3a shows an example of an Si-Pt-Teflon texture in which the plastron regeneration mechanism is self-limiting. The textures were immersed in deionized water and imaged at an angle of $45^{\circ}$ with yellow light reflecting off the air-water interface as shown in Figure S1. For example, the first frame in Figure 3b shows an image of an immersed silicon microtexture (150 $\mu \mathrm{m}$ depth, $50 \mu \mathrm{m}$ pillar width, $100 \mu \mathrm{m}$ pitch corresponding to $\left.\phi_{s}=0.196, r=3.356\right)$ which has been partially impregnated by water. The plastron filled region reflects yellow light via total internal reflection at the air-water interface and appears golden in color, while the other wetted regions appear dark due to the absence of air within the texture. The subsequent frames in Figure 3b show plastron recovery after addition of a few drops of 30 wt.\% hydrogen peroxide to the solution (Final $\mathrm{H}_{2} \mathrm{O}_{2}$ concentration in solution $=4.5 \%$ wt.). Defects in the hydrophobic coating allow the dissolved hydrogen peroxide to come in contact with the $\mathrm{Pt}$ 

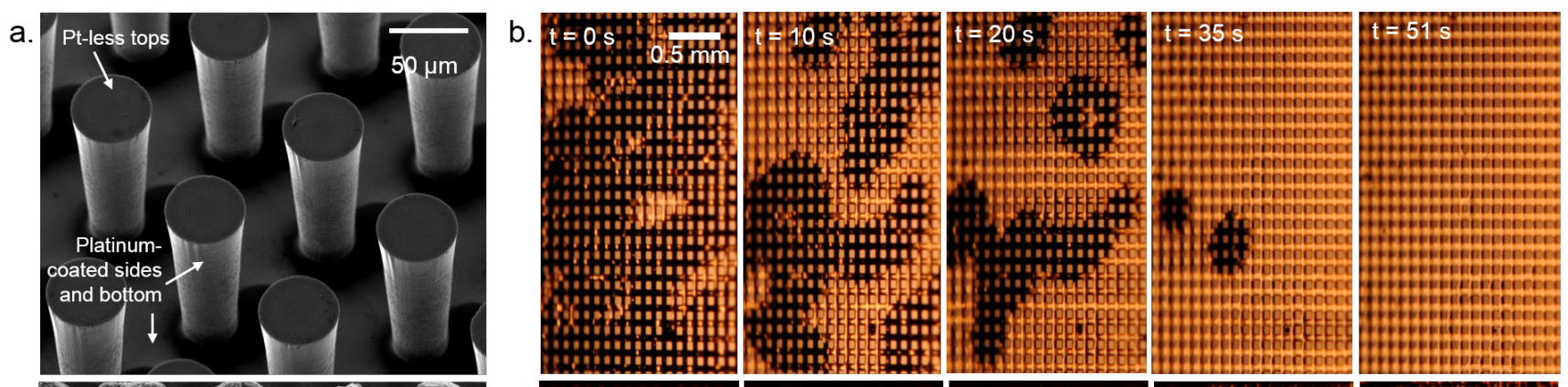

c.

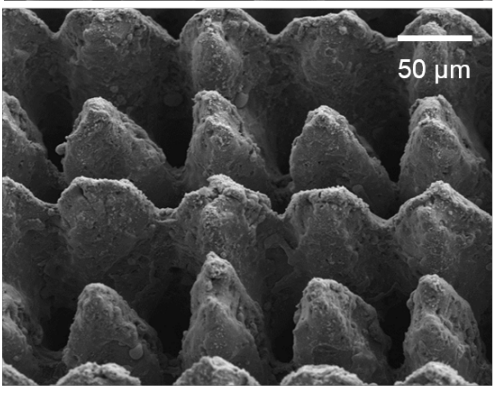

d. $t=0 \mathrm{~s}$

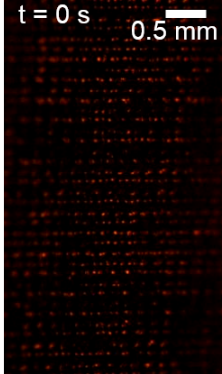

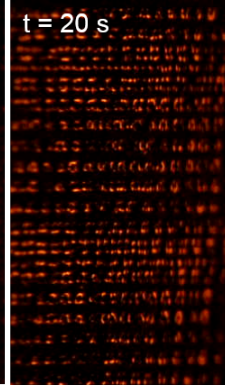
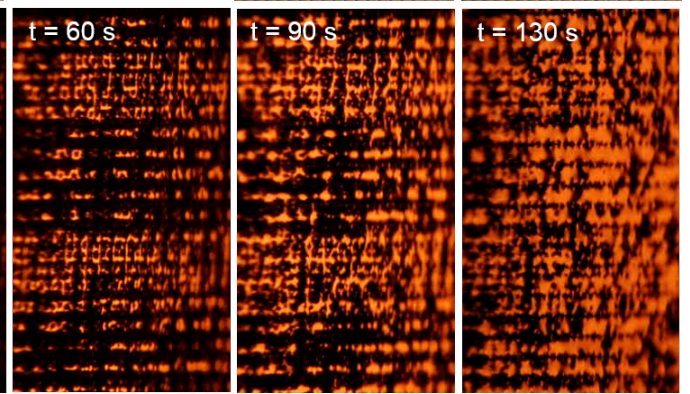

Figure 3: (a) Micrograph of silicon texture with platinum coating on the sides and bottom of the texture, and Teflon coating on the entire texture (Si-Pt-Teflon); (b) Plastron recovery on Si-Pt-Teflon texture underwater at 4.5 wt. $\% \mathrm{H}_{2} \mathrm{O}_{2}$; (c) Micrograph of laser textured aluminum pillars coated with $\mathrm{MnO}_{2}$ and Fluorosilane (Al- $\mathrm{MnO}_{2}$-Fluorosilane); (d) Plastron recovery underwater on $\mathrm{Al}-\mathrm{MnO}_{2}$-Fluorosilane texture at 1.5 wt. $\% \mathrm{H}_{2} \mathrm{O}_{2}$

catalyst in between the pillars of the texture and the resulting decomposition reaction causes oxygen gas to nucleate within the microtexture. Figure $3 \mathrm{~b}$ shows the time evolution of a Si-Pt-Teflon surface transitioning from a partially-wetted state to a fully-recovered plastron state. Supplementary Video S1 shows the process of plastron nucleation and lateral spreading through the texture. Experiments were also performed on silicon micropillars whose tops were not stripped of platinum catalyst (See Supplementary Figure S2) and it was found that plastron recovery is possible in these cases too. We believe that this is possible because the nucleation of gas is favored at the sharp corners at the bottom of the texture, even though there is catalyst at the top. We also note that Si micropillars fabricated using the Bosch RIE process also have a nanoscale texture which can promote plastron recovery. Hence, further tests were conducted using smooth-walled micropillars made of SU8 photoresist (See Supplementary Figure S4). It was observed that smooth-walled micropillar textures also show plastron recovery similar to the dry-etched Si micropillars, further supporting our 
observation that nanoscale roughness is not necessary for plastron recovery.

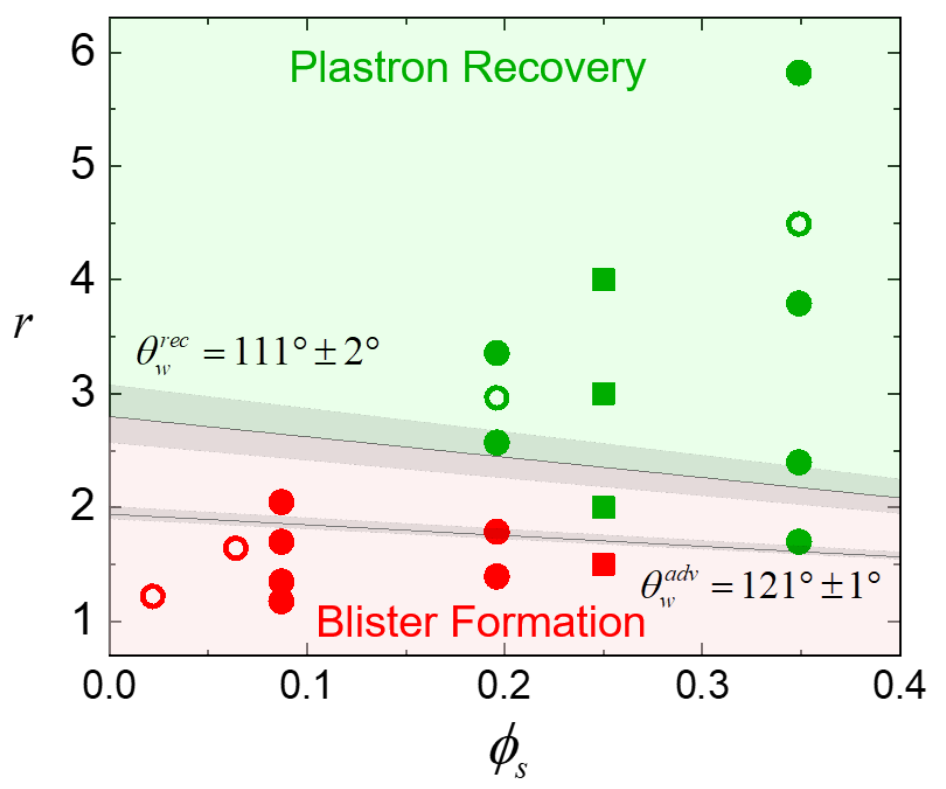

Figure 4: Regime map for plastron reinflation experiments on Si-Pt-Teflon surfaces with varying texture parameters. The colors indicate whether plastron recovery was observed: plastron recovery (green) and blisters (red); The shape of the markers indicate the shape of the posts: cylindrical pillars (circles), square cylindrical pillars (square). The marker filling indicates whether the catalyst was deposited on the pillar tops: no catalyst on top (filled), catalyst on top (hollow)

Figure 4 shows a regime map classifying the silicon micro-pillar experiments into two categories based on whether plastron spreading or blister formation was favored based on the texture parameters characterizing each surface. The receding contact angle of a water droplet on a Teflon coated smooth silicon wafer was measured to be $\theta_{w}^{r e c}=111^{\circ} \pm 2^{\circ}$ and this value has been used to plot the recovery condition given by Equation 6. The shaded region around the theoretical prediction line indicates the sensitivity of Equation 6 to a receding contact angle variation of $\pm 2^{\circ}$. It can be seen from both the theory and the experiments that textures with larger roughness and higher solid fraction favor plastron recovery, while those with lower roughness and smaller solid fraction promote blister formation. We also see that the textures which have catalyst on top of the pillars favor plastron recovery as well. Although it has been reported in previous studies that the presence of a nano-Cassie state is 
necessary for plastron recovery, ${ }^{29,36}$ we observe that satisfying the thermodynamic condition for plastron gas spreading alone is sufficient to allow for plastron recovery on microtextures even without a nanoscale texture to hold a stable plastron. This is also in agreement with the observation by Forsberg et. al., ${ }^{37}$ who reported plastron recovery on smooth-walled polymer pillars. It can be observed that a few textures show recovery even though they lie below the theoretically condition for plastron recovery. This can be explained by the fact that these textures can transition from a metastable Wenzel state to a stable Cassie state if they satisfy Equation 6 with $\theta_{w}^{r e c}=\theta_{w}^{y o u n g}$, the Young contact angle for Teflon. This curve would lie in between the theoretical curves for $\theta_{w}^{a d v}$ and $\theta_{w}^{r e c}$ shown in Figure 4, and define the bi-stable region where the Wenzel state is metastable. ${ }^{26}$ In underwater Wenzel-to-Cassie transitions, the energy barrier between the Cassie and Wenzel states in the bistable region can be overcome by the gas over-pressure in the plastron due to continuous generation of gas. We believe that this thermodynamic condition derived assuming a simple patterned texture is a necessary but not a sufficient condition for plastron recovery. While this condition is an important global factor, there are various other local factors which can also influence the dynamics of plastron spreading such as the pillar breakthrough pressure, ${ }^{29}$ contact of gas meniscus with pillars in the direction of spreading, ${ }^{37}$ contact line pinning, and texture re-entrance. ${ }^{56}$

In order to make this method scalable for large scale drag reduction applications, catalytically active textures were fabricated on textured aluminum using an inexpensive catalyst, manganese dioxide. Flat polished aluminum plates were laser textured, electroplated with manganese dioxide, and conformally coated with a fluorinated silane to obtain catalytically active superhydrophobic textures (Details of the procedure are explained in the experimental section). Figure $3 \mathrm{c}$ shows a micrograph of a so-called egg-crate ${ }^{57}$ aluminum microtexture coated with $\mathrm{MnO}_{2}$ and silane. The Wenzel roughness of this texture was calculated from the surface area measured using an optical profilometer and corresponds to $r=5.1$. The electroplated $\mathrm{MnO}_{2}$ deposit has both microscale and nanoscale features as can be seen in 
Supplementary Figure S3. Since the texture has a dual scale roughness, it is expected that the nano-Cassie state can be supported in the nanofeatures of the texture even after impregnation at the micro-scale. ${ }^{36}$ Supplementary Video S2 shows the inflation of plastron within the egg-crate aluminum microtexture. In order to ensure maximum water impregnation in the texture, the samples were immersed into boiled deionised water under vacuum for 12 hours before the introduction of hydrogen peroxide. Figure 3d shows the process of plastron recovery on such a texture at a hydrogen peroxide concentration of $1.5 \mathrm{wt} . \%$. Note that the $\mathrm{MnO}_{2}$ coating was not removed from the tops of the aluminum pyramids in this experiment; however, plastron recovery was still favored over blister formation. This is in agreement with our previous observation on silicon pillars with platinum-coated tops.

In all of the experiments for which plastron recovery was observed, the gas spreads laterally along the texture until the contact line becomes sufficiently pinned to prevent further advancement. When the contact line is unable to de-pin further, the gas breaks through the top of the pillars and forms a hemiwicking blister. At this point the gas generated within the texture is preferentially channeled into the hemiwicking blister which has a low Laplace pressure due to the large radius of curvature of the blister. This stops the connected plastron from spreading further into other wetted parts of the texture. Hence, it is important to fabricate surface textures with high plastron connectivity and low pinning defects for complete plastron recovery.

To measure the drag reduction performance and slip recovery capabilities of these plastron promoting textures, aluminum rotors with catalytically active textures were tested in a Taylor-Couette cell under turbulent flow conditions. ${ }^{16}$ Cylindrical rotors $\left(R_{\text {rotor }}=14 \mathrm{~mm}\right)$ were textured by machining microgrooves onto the surface in both the circumferential and longitudinal directions to obtain a regular array of square micropillars of width $100 \mu \mathrm{m}$, height $200 \mu \mathrm{m}$, and spacing $100 \mu \mathrm{m}\left(r=2, \phi_{s}=0.25\right)$. The rotor was then electroplated with $\mathrm{MnO}_{2}$ and hydrophobized using a fluorinated silane in a similar manner to the flat laser-textured aluminum samples. Figure 5a shows a micrograph of this texture; where 
the micro-deposits on the pillars correspond to $\mathrm{MnO}_{2}$ electroplated on the texture, while Figure 5b shows a droplet of water sitting on the superhydrophobic surface of the textured aluminum rotor. All drag reduction experiments were performed under fully turbulent flow conditions in a custom-fabricated Taylor-Couette Cell (shown in Figure 5c) at Re =47,000, where the Reynolds number is calculated as $\operatorname{Re}=R_{i} \Omega \Delta R / \nu$. Here $R_{i}$ is the rotor radius, $\Delta R$ is the cell gap, $\Omega$ is the angular velocity of the rotor $\left(R_{\text {rotor }}\right)$, and $\nu$ is the kinematic viscosity of the working fluid. The mean value of the frictional wall shear stress $\left(\tau_{w}\right)$ on the rotor is defined as $\tau_{w}=\bar{T} / 2 \pi R_{i}^{2} L$ where $\bar{T}$ and $L$ are the averaged torque experienced by the rotor and the length of the rotor respectively. The percentage drag reduction is defined as $D R=100 \times\left(\tau_{\text {Smooth }}-\tau_{\text {Cassie }}\right) / \tau_{\text {Smooth }}$, where $\tau_{\text {Smooth }}$ is the shear stress on an untextured cylindrical rotor and $\tau_{\text {Cassie }}$ is the shear stress on the patterned rotor with a fully inflated plastron. Note that the solid fraction $\phi_{s}$ of the grooved rotor is high $\left(\phi_{s}=0.25\right)$ to promote plastron regeneration (See Figure 4), but the experimentally measured turbulent drag reduction is only about $3 \%$ compared to a smooth untextured hydrophilic rotor at the same Reynolds number of $\operatorname{Re}=47,000$. However, this rotor can still be used to evaluate the effectiveness of the plastron recovery method by comparing the torques on the rotor in its original plastron inflated state (Cassie), its wetted state (Wenzel) and its final recovered state.

The plastron recovery tests were performed in three stages as shown in Figure 5e. The plastron is initially tested for its stability by running a long test (4 hours) under air-saturated water conditions. There is minimal change in the shear stress, $\tau_{\text {Cassie }}$, as the plastron is stable during this time period. A wetting transition was then artificially induced by flushing de-aerated water through the system until the shear stress rises and reaches a new steady state value corresponding to a fully wetted Wenzel state, $\tau_{\text {Wenzel }}$. Visual inspection of the textured rotor in the cell also showed almost complete loss of the silvery plastron film. The rotor was maintained in this state for a few minutes before introducing hydrogen peroxide into the system. $1 \mathrm{ml}$ of $7.5 \mathrm{wt} \%$ hydrogen peroxide was introduced into the cell over a 
a.

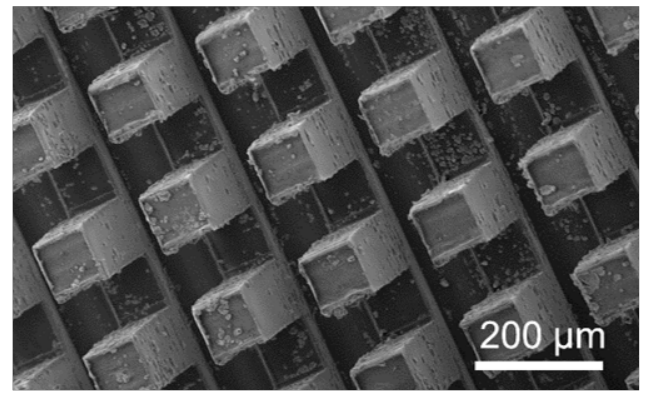

b.

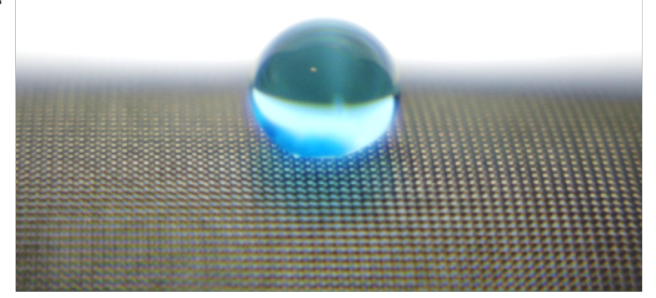

d.

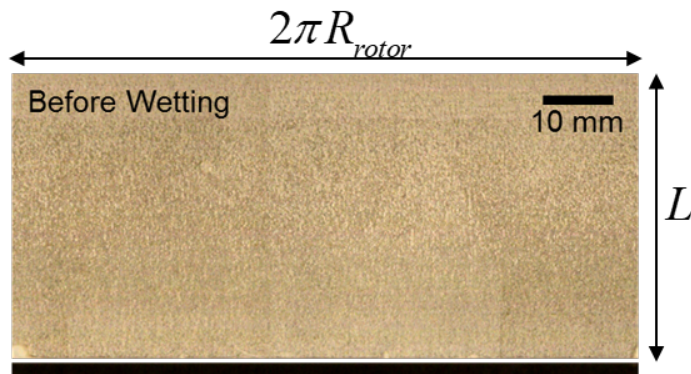

After Wetting
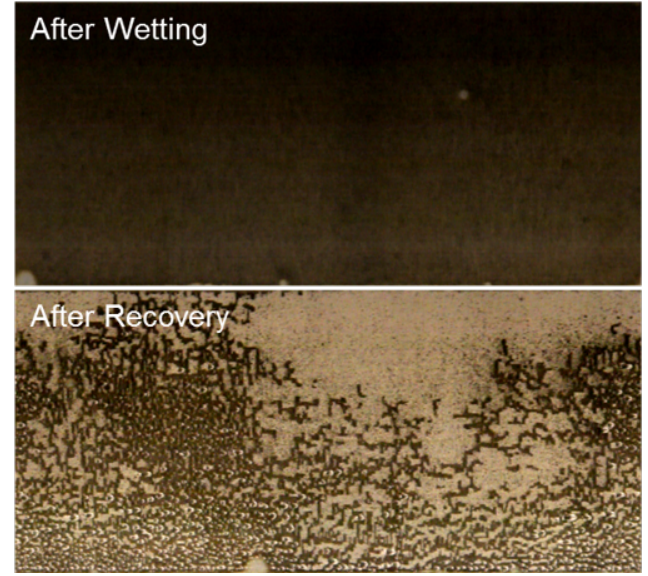

c.

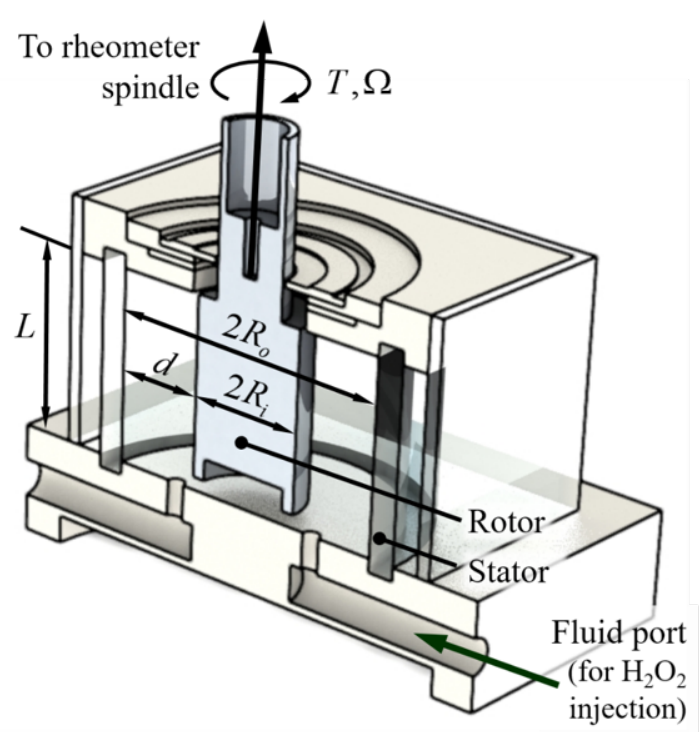

e.

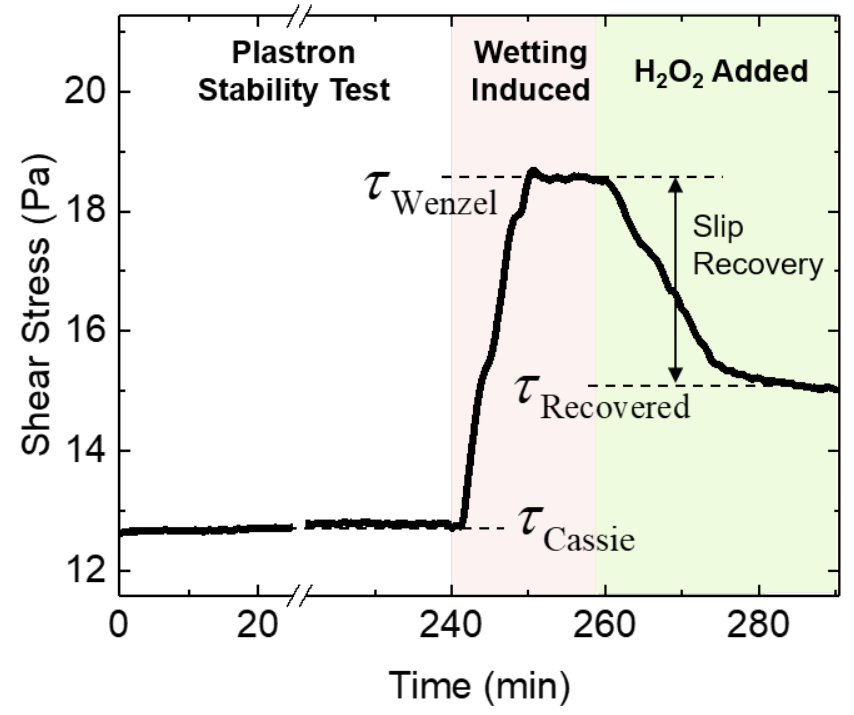

Figure 5: (a) Micrograph of square pillars (width $100 \mu \mathrm{m}$, height $200 \mu \mathrm{m}$, and spacing 100 $\mu \mathrm{m})$ machined on an aluminum rotor of radius $R_{i}=14 \mathrm{~mm}$ and coated with $\mathrm{MnO}_{2}$ and a fluorinated silane; (b) Drop of water $(\mathrm{V} \sim 12 \mu \mathrm{L})$ sitting on the hydrophobized machined rotor used for plastron recovery $\left(\theta_{a d v}=159 \pm 2, \theta_{\text {rec }}=109 \pm 5\right)$; (c) Schematic of the TaylorCouette cell used for drag reduction measurements with fluid port for $\mathrm{H}_{2} \mathrm{O}_{2}$ injection; (d) Unwrapped views of the cylindrical surface of the rotor before plastron failure, after plastron failure, and after partial plastron recovery. This image was produced by imaging the rotating rotor at constant speed, and stitching together vertical sections of the rotor captured across one entire rotation. (e) Plot of the average wall shear stress, $\tau_{w}$, experienced by the rotor in the Taylor-Couette cell at $\mathrm{Re}=47,000$ during the initial period of drag reduction (Cassie state), plastron failure (Wenzel state) and plastron recovery (partial Cassie) stages. 
period of 5 mins to increase the $\mathrm{H}_{2} \mathrm{O}_{2}$ bulk concentration up to $1.3 \times 10^{-5} \mathrm{~mol} \mathrm{ml}^{-1}$. The average shear stress exerted on the inner rotor is observed to decrease slowly and reaches a steady state value, $\tau_{\text {Recovered }}$, over a period of 20 min after which any additional oxygen produced only contributed to blister formation on the rotor. The plastron recovery is observed to be patchy in most parts due to pinning defects as can be seen in Supplementary Figure S5. Figure 5d shows "unwrapped" views of the rotor in its Cassie state, Wenzel state and after partial recovery (reconstructed from a sequence of images). Although the plastron recovery is imperfect, the presence of the air layer contributes to improving the average level of wall slip induced on the rotor surface. The percentage slip recovery is calculated as $S R=100 \times\left(\tau_{\text {Wenzel }}-\tau_{\text {Cassie }}\right) / \tau_{\text {Recovered }}$, where $\tau_{\text {Wenzel }}$ is the torque on the completely wetted rotor and $\tau_{\text {Recovered }}$ is the torque on the rotor after plastron recovery. The average slip recovery percentage over four repeated experiments was found to be $51 \pm 14 \%$.

It can be estimated from the rotor texture parameters that $6.1 \times 10^{-7} \mathrm{~mol}$ of oxygen is required for every square centimeter of rotor surface for plastron recovery to occur over the complete surface area of the rotor assuming near atmospheric pressure conditions and a texture depth of $\mathrm{H}=200 \mu \mathrm{m}$. However, since all the hydrogen peroxide injected does not come into contact with the catalyst surface and thus does not contribute to plastron recovery, it is necessary to inject an amount of hydrogen peroxide, $n_{\mathrm{H}_{2} \mathrm{O}_{2}}^{\text {injected }}$, higher than the required stoichiometric quantity, $n_{\mathrm{H}_{2} \mathrm{O}_{2}}^{\text {utized }}$, in order to achieve a significant rate of plastron recovery. The maximum efficiency of chemical regeneration of plastron can be calculated as $\eta_{\max }=n_{\mathrm{H}_{2} \mathrm{O}_{2}}^{\text {utilized }} / n_{\mathrm{H}_{2} \mathrm{O}_{2}}^{\text {injected }}$, and it was found to be $1.1 \%$ in the current configuration. Since the plastron recovery is partial, this efficiency must also be multiplied by the percentage area recovered to get the effective efficiency. For example, in Figure 5d, the percentage of the wetted area over which plastron recovery is observed is only estimated to be $58 \%$, and hence the efficiency $\eta=0.6 \%$. The plastron recovery coverage could be increased by incorporating a dual-scaled texture like that in the laser textured aluminum substrates, which allows for the gas to spread more easily through the nanotexture without pinning, thus reducing contact 
angle hysteresis. The mass transfer efficiency could be also potentially improved by injecting the hydrogen peroxide directly into the boundary layer from within a porous surface.

\section{Conclusion}

We have demonstrated a novel chemical method to replenish a surface air layer or plastron in-situ on superhydrophobic textures that are fully immersed underwater using catalytic decomposition of hydrogen peroxide. We also showed that plastron spreading is possible on microtextures without a nano-Cassie state by performing experiments on silicon microtextures with platinum catalyst without a second level of nano-texture. Our thermodynamic framework for predicting recovery on hydrophobic textures can be used to design textures with optimal roughness and surface chemistry functionalization for plastron recovery. We also demonstrate the scalability of this method by fabricating a suitable 'egg crate' structure on laser-textured aluminum surfaces with an inexpensive manganese dioxide catalyst and demonstrating plastron recovery on this surface. Finally we have performed fluid frictional drag measurements on a microtextured aluminum rotor loaded with $\mathrm{MnO}_{2}$ catalyst. Skin friction measurements in a turbulent Taylor-Couette flow showed that it is possible to obtain an average slip recovery of $51 \pm 14 \%$ from the texture's fully wetted state when hydrogen peroxide was added to the system. We anticipate that these catalytically-active drag-reducing surface microtextures can be scaled-up further and implemented in the marine environemnt to extend the stable lifetimes of frictional drag reduction and anti-corrosion.

\section{Experimental Methods}

\section{Fabrication of Silicon Micropillars with Catalyst-free Tops}

We fabricated microtextures with selective catalyst coverage on silicon wafers using image reversal photolithography, reactive ion etching (RIE), e-beam evaporation of catalyst onto 
the pillars, and subsequent lift-off of the catalyst from the tops of the micropillars. 6" Silicon wafers (University wafers) were first coated with the image reversal photoresist, AZ5214, and an array of circular pillars of photoresist was created on the wafer by using image reversal photolithography. RIE (STS Pegasus) was then used to etch the exposed Si in the photoresist mask to create micropillars in the silicon. The depth of the pillars was controlled by adjusting the RIE process time. Platinum was uniformly deposited onto the pillars using e-beam evaporation. The catalyst on the tops of the micropillars was subsequently removed using a lift-off procedure. These pillars were then spin-coated with Teflon AF solution (1 wt.\%, Chemours) at $3000 \mathrm{rpm}$ to render the surface chemistry of the microtexture array hydrophobic. Figure 3a shows a micrograph of a Si-Pt-Teflon texture with $150 \mu \mathrm{m}$ depth, and Pt-less tops. The lift-off step was omitted for testing the samples in which the catalyst was retained on the tops of the pillars and these are shown in Figure S2. The dimensions of these pillars are given in Table S1 in the supplementary material. Smooth-walled micropillar arrays were fabricated using photolithography with SU8 photoresist on Pt-coated Si wafers.

\section{Fabrication of Aluminium Micropillars with Catalyst Coating}

We fabricated catalyst-impregnated superhydrophobic aluminium microtextures by using laser texturing, electroplating and silanization. An egg-crate microtexture $(r=5.1)$ was patterned onto flat sheets of Aluminum (Al 6106) by scribing equally spaced lines (pitch = $75 \mu \mathrm{m}$ ) in the horizontal and vertical directions using a laser scriber (Electrox Scriba E-Box, $200 \mathrm{~mm} / \mathrm{s}, 20$ repetitions). This surface was then dipped into $5 \mathrm{wt} \%$ sodium hydroxide for 45 s to remove any organic contaminants. The substrate was then electroplated with manganese dioxide by using anodic electrolytic deposition. ${ }^{58}$ The substrate and counter electrode (a flat Al plate cleaned using the sodium hydroxide protocol described earlier) were dipped into 100 $\mathrm{ml}$ of $0.05 \mathrm{M}$ Manganese acetate solution with a gap of $20 \mathrm{~mm}$ between them. The solution was maintained at $60{ }^{\circ} \mathrm{C}$ and stirred using a stir bar at $60 \mathrm{rpm}$. Electrolytic deposition was performed at $5 \mathrm{~mA} / \mathrm{cm}^{2}$ for $5 \mathrm{~min}$, and then the electrodes were immediately removed 
from the solution and dried at $100{ }^{\circ} \mathrm{C}$ in air. Fresh solutions of $\mathrm{Mn}\left(\mathrm{CH}_{3} \mathrm{COO}\right)_{2}$ were used for every new deposition. Figure $\mathrm{S} 3$ shows $\mathrm{MnO}_{2}$ electrodeposited on a flat Al substrate under similar deposition conditions. The electroplated substrates were then cleaned using a plasma cleaner (Harrick) for 5 mins and placed into a glass beaker for silane chemical vapor deposition. An open vial with $3 \mathrm{ml}$ of a fluorinated silane (Trichloro $(1 \mathrm{H}, 1 \mathrm{H}, 2 \mathrm{H}, 2 \mathrm{H}-$ perfluorooctyl)silane) was placed into the glass beaker along with the other substrates. The

beaker was then covered with an aluminum foil and placed on a hotplate at $120{ }^{\circ} \mathrm{C}$ for 3 hours. The substrates were then rinsed with isopropanol to remove excess silane and dried in air, to obtain a superhydrophobic texture with $\left(\theta_{a d v}=162^{\circ} \pm 3^{\circ}, \theta_{r e c}=148^{\circ} \pm 4^{\circ}\right)$

\section{Imaging of plastron recovery}

The substrates were immersed into a quartz cell $(2 \mathrm{~L} \times 1 \mathrm{~W} \mathrm{~W} \times 2 \mathrm{H}$, ramé-hart) containing $30 \mathrm{ml}$ of de-ionized (DI) water and supported at approximately $45^{\circ}$ to the horizontal plane. A microscope (ZEISS Axio Zoom.V16) was used to observe the plastron at high magnification. A spot light source (Dolan-Jenner MI-150) was used to illuminate the plastron at an angle greater than the critical angle for total internal reflection $\left(\theta_{c}=48.75^{\circ}\right)$ to visualize the airwater interface as shown in Figure S1. The plastron that is initially present on the substrate after immersion into DI water was removed from the substrates by applying suction/pressure at the surface using a pipette. To trigger plastron recovery, 1-3 $\mathrm{ml}$ of $30 \mathrm{wt} . \%$ hydrogen peroxide (inhibited hydrogen peroxide, Sigma Aldrich) was gently added to the water and allowed to diffuse to the surface. Videos were then recorded using a CCD camera at $24 \mathrm{fps}$.

\section{Drag Reduction Experiments}

Drag reduction experiments were performed on a controlled stress rheometer (TA Instruments AR-G2) using a bespoke Taylor-Coutte cell with rotor radius $14 \mathrm{~mm}$, cell gap 20 $\mathrm{mm}$ and rotor length $42 \mathrm{~mm},{ }^{16}$ and a fluid inlet port to inject hydrogen peroxide into the cell during the experiment as shown in Figure 5c. In this Taylor-Coutte cell, transition to 
fully turbulent flow occurs at $\operatorname{Re}>10,000$. Microgrooves were machined onto the rotor (Al 6061-T6) using a CNC lathe (Burlington Machine, Wilmington, MA). The rotor was then electroplated in manganese acetate solution $\left(0.05 \mathrm{M}, 60^{\circ} \mathrm{C}\right.$, stirring at $\left.60 \mathrm{rpm}\right)$ with a cylindrical counter electrode at a current density of $5 \mathrm{~mA} / \mathrm{cm}^{2}$ for $5 \mathrm{~min}$. It was then dried and plasma cleaned in air for 5 min before depositing silane using the same procedure as the flat aluminum plates.

\section{Acknowledgement}

The authors thank KFUPM (King Fahd University of Petroleum and Minerals) for their financial support. The authors also thank the staff at MTL (Microsystems Technology Laboratories, MIT) for their input in silicon pillar fabrication, the 'Photovoltaics Research Laboratory' (Prof. Tonio Bounassisi) for the laser scribing facility, and Dr. Siddarth Srinivasan for the insightful discussions.

\section{Supporting Information Available}

- Supplementary Information : File contains figures on plastron imaging, extra micrographs of surfaces and a table of dimensions of all the silicon textures used.

- Video S1: Video of plastron recovery on a Silicon pillar array.

- Video S2: Video of plastron recovery on a Aluminium laser textured surface.

\section{References}

(1) Drelich, J.; Marmur, A. Physics and applications of superhydrophobic and superhydrophilic surfaces and coatings. Surface Innovations 2014, 2, 211-227. 
(2) Park, K. C.; Chhatre, S. S.; Srinivasan, S.; Cohen, R. E.; McKinley, G. H. Optimal design of permeable fiber network structures for fog harvesting. Langmuir 2013, 29, $13269-13277$.

(3) Bengaluru Subramanyam, S.; Kondrashov, V.; Rühe, J.; Varanasi, K. K. Low Ice Adhesion on Nano-Textured Superhydrophobic Surfaces under Supersaturated Conditions. ACS Applied Materials and Interfaces 2016, 8, 12583-12587.

(4) Arnott, J.; Wu, A. H. F.; Vucko, M. J.; Lamb, R. N. Marine antifouling from thin air. Biofouling 2014, 30, 1045-1054.

(5) Feng, X. J.; Jiang, L. Design and Creation of Superwetting/Antiwetting Surfaces. Advanced Materials 2006, 18, 3063-3078.

(6) Butt, H. J.; Semprebon, C.; Papadopoulos, P.; Vollmer, D.; Brinkmann, M.; Ciccotti, M. Design principles for superamphiphobic surfaces. Soft Matter 2013, 9, 418428.

(7) Wong, T. S.; Sun, T.; Feng, L.; Aizenberg, J. Interfacial materials with special wettability. MRS Bulletin 2013, 38, 366-371.

(8) Cassie, A. B. D.; Baxter, S. Wettability of porous surfaces. Transactions of the Faraday Society 1944, 40, 546-551.

(9) Tian, Y.; Su, B.; Jiang, L. Interfacial Material System Exhibiting Superwettability. Advanced Materials 2014, 26, 6872-6897.

(10) George, J. E.; Chidangil, S.; George, S. D. Recent Progress in Fabricating Superaerophobic and Superaerophilic Surfaces. Advanced Materials Interfaces 2017, 4, 1601088.

(11) Thorpe, W. H. Plastron Respiration in Aquatic Insects. Biological Reviews 1950, 25, $344-390$. 
(12) Flynn, M. R.; Bush, J. W. M. Underwater breathing: the mechanics of plastron respiration. Journal of Fluid Mechanics 2008, 608, 275-296.

(13) Barthlott, W.; Schimmel, T.; Wiersch, S.; Koch, K.; Brede, M.; Barczewski, M.; Walheim, S.; Weis, A.; Kaltenmaier, A.; Leder, A.; Bohn, H. F. The salvinia paradox: superhydrophobic surfaces with hydrophilic pins for air retention under water. Advanced Materials 2010, 22, 2325-2328.

(14) Wang, P.; Qiu, R.; Zhang, D.; Lin, Z.; Hou, B. Fabricated super-hydrophobic film with potentiostatic electrolysis method on copper for corrosion protection. Electrochimica Acta 2010, 56, 517-522.

(15) Rothstein, J. P. Slip on Superhydrophobic Surfaces. Annual Review of Fluid Mechanics 2010, 42, 89-109.

(16) Srinivasan, S.; Kleingartner, J. A.; Gilbert, J. B.; Cohen, R. E.; Milne, A. J. B.; McKinley, G. H. Sustainable Drag Reduction in Turbulent Taylor-Couette Flows by Depositing Sprayable Superhydrophobic Surfaces. Physical Review Letters 2015, 114, 014501.

(17) Bobji, M. S.; Kumar, S. V.; Asthana, A.; Govardhan, R. N. Underwater sustainability of the "Cassie" state of wetting. Langmuir 2009, 25, 12120-12126.

(18) Samaha, M. A.; Tafreshi, H. V.; Gad-el Hak, M. Influence of flow on longevity of superhydrophobic coatings. Langmuir 2012, 28, 9759-9766.

(19) Bocquet, L.; Lauga, E. A smooth future? Nature Materials 2011, 10, 334-337.

(20) Govardhan, R. N.; Srinivas, G. S.; Asthana, A.; Bobji, M. S. Time dependence of effective slip on textured hydrophobic surfaces. Physics of Fluids 2009, 21, 052001.

(21) Lyu, S.; Nguyen, D. C.; Kim, D.; Hwang, W.; Yoon, B. Experimental drag reduction study of super-hydrophobic surface with dual-scale structures. Applied Surface Science 2013, 286, 206-211. 
(22) Wenzel, R. N. Resistance of solid surfaces to wetting by water. Industrial \& Engineering Chemistry 1936, 28, 988-994.

(23) Vrancken, R. J.; Kusumaatmaja, H.; Hermans, K.; Prenen, A. M.; Pierre-Louis, O.; Bastiaansen, C. W. M.; Broer, D. J. Fully reversible transition from Wenzel to CassieBaxter states on corrugated superhydrophobic surfaces. Langmuir 2010, 26, 3335-3341.

(24) Manukyan, G.; Oh, J. M.; Van Den Ende, D.; Lammertink, R. G. H.; Mugele, F. Electrical switching of wetting states on superhydrophobic surfaces: A route towards reversible Cassie-to-Wenzel transitions. Physical Review Letters 2011, 106, 014501.

(25) Krupenkin, T. N.; Taylor, J. A.; Wang, E. N.; Kolodner, P.; Hodes, M.; Salamon, T. R. Reversible wetting-dewetting transitions on electrically tunable superhydrophobic nanostructured surfaces. Langmuir 2007, 23, 9128-9133.

(26) Li, Y.; Quéré, D.; Lv, C.; Zheng, Q. Monostable superrepellent materials. Proceedings of the National Academy of Sciences 2017, 114, 3387-3392.

(27) Boreyko, J. B.; Chen, C. H. Restoring superhydrophobicity of lotus leaves with vibration-induced dewetting. Physical Review Letters 2009, 103, 174502.

(28) Stamatopoulos, C.; Schutzius, T. M.; Köppl, C. J.; El Hayek, N.; Maitra, T.; Hemrle, J.; Poulikakos, D. On the shedding of impaled droplets: The role of transient intervening layers. Scientific Reports 2016, 6, 18875.

(29) Lee, C.; Kim, C.-J. Underwater Restoration and Retention of Gases on Superhydrophobic Surfaces for Drag Reduction. Physical Review Letters 2011, 106, 014502.

(30) Lloyd, B. P.; Bartlett, P. N.; Wood, R. J. K. Active Gas Replenishment and Sensing of the Wetting State in a Submerged Superhydrophobic Surface. Soft Matter 2017, 13, $1413-1419$. 
(31) Lee, J.; Yong, K. Combining the lotus leaf effect with artificial photosynthesis: regeneration of underwater superhydrophobicity of hierarchical $\mathrm{ZnO} / \mathrm{Si}$ surfaces by solar water splitting. NPG Asia Materials 2015, 7, e201.

(32) Vakarelski, I. U.; Patankar, N. A.; Marston, J. O.; Chan, D. Y.; Thoroddsen, S. T. Stabilization of Leidenfrost vapour layer by textured superhydrophobic surfaces. Nature 2012, 489, 274-277.

(33) Saranadhi, D.; Chen, D.; Kleingartner, J. A.; Srinivasan, S.; Cohen, R. E.; McKinley, G. H. Sustained drag reduction in a turbulent flow using a low-temperature Leidenfrost surface. Science Advances 2016, 2, e1600686.

(34) Hof, B. Fluid dynamics: Water flows out of touch. Nature 2017, 541, 161-162.

(35) Ling, H.; Srinivasan, S.; Golovin, K.; McKinley, G. H.; Tuteja, A.; Katz, J. Highresolution velocity measurement in the inner part of turbulent boundary layers over super-hydrophobic surfaces. Journal of Fluid Mechanics 2016, 801, 670-703.

(36) Verho, T.; Korhonen, J. T.; Sainiemi, L.; Jokinen, V.; Bower, C.; Franze, K.; Franssila, S.; Andrew, P.; Ikkala, O.; Ras, R. H. A. Reversible switching between superhydrophobic states on a hierarchically structured surface. Proceedings of the National Academy of Sciences 2012, 109, 10210-10213.

(37) Forsberg, P.; Nikolajeff, F.; Karlsson, M. Cassie-Wenzel and Wenzel-Cassie transitions on immersed superhydrophobic surfaces under hydrostatic pressure. Soft Matter $\mathbf{2 0 1 1}$, 7, 104-109.

(38) Du, P.; Wen, J.; Zhang, Z.; Song, D.; Ouahsine, A.; Hu, H. Maintenance of air layer and drag reduction on superhydrophobic surface. Ocean Engineering 2017, 130, 328-335.

(39) de Maleprade, H.; Clanet, C.; Quéré, D. Spreading of Bubbles after Contacting the 
Lower Side of an Aerophilic Slide Immersed in Water. Physical Review Letters 2016, 11\%, 094501.

(40) Davis, A. M.; Lauga, E. Geometric transition in friction for flow over a bubble mattress. Physics of Fluids 2009, 21, 011701.

(41) Ling, H.; Katz, J.; Fu, M.; Hultmark, M. Effect of Reynolds number and saturation level on gas diffusion in and out of a superhydrophobic surface. Physical Review Fluids 2017, 2, 124005.

(42) Vakarelski, I. U.; Chan, D. Y. C.; Marston, J. O.; Thoroddsen, S. T. Dynamic air layer on textured superhydrophobic surfaces. Langmuir 2013, 29, 11074-11081.

(43) Lin, C. C.; Smith, F. R.; Ichikawa, N.; Baba, T.; Itow, M. Decomposition of Hydrogen Peroxide in Aqueous Solutions at Elevated Temperatures. International Journal of Chemical Kinetics 1991, 23, 971-987.

(44) May, D. W. Catalase, A New Enzym of General Occurrence. Science 1901, 14, 815-816.

(45) McKee, D. W. Catalytic decomposition of hydrogen peroxide by metals and alloys of the platinum group. Journal of Catalysis 1969, 14, 355-364.

(46) Liebhafsky, H. A. The Catalytic Decomposition of Hydrogen Peroxide By the IodineIodide Couple At 25․ Journal of the American Chemical Society 1932, 54, 1792-1806.

(47) Roy, C. B. Catalytic Decomposition of Hydrogen Peroxide on some Oxide Catalysts. Journal of Catalysis 1968, 12, 129-133.

(48) Sanchez, S.; Solovev, A. A.; Mei, Y. F.; Schmidt, O. G. Dynamics of Biocatalytic Microengines Mediated by Variable Friction Control. Journal of American Chemical Society 2010, 132, 13144-13145. 
(49) Wang, Y.; Hernandez, R. M.; Bartlett, D. J.; Bingham, J. M.; Kline, T. R.; Sen, A.; Mallouk, T. E. Bipolar electrochemical mechanism for the propulsion of catalytic nanomotors in hydrogen peroxide solutions. Langmuir 2006, 22, 10451-10456.

(50) Jones, P. R.; Hao, X.; Cruz-Chu, E. R.; Rykaczewski, K.; Nandy, K.; Schutzius, T. M.; Varanasi, K. K.; Megaridis, C. M.; Walther, J. H.; Koumoutsakos, P.; Espinosa, H. D.; Patankar, N. A. Sustaining dry surfaces under water. Scientific Reports 2015, 5, 12311.

(51) Marmur, A. Underwater Superhydrophobicity: Theoretical Feasibility. Langmuir 2006, 22, 1400-1402.

(52) Grossmann, S.; Lohse, D.; Sun, C. High-Reynolds Number Taylor-Couette Turbulence. Annual Review of Fluid Mechanics 2016, 48, 53-80.

(53) Shi, C.; Cui, X.; Zhang, X.; Tchoukov, P.; Liu, Q.; Encinas, N.; Paven, M.; Geyer, F.; Vollmer, D.; Xu, Z.; Butt, H.-J.; Zeng, H. Interaction between Air Bubbles and Superhydrophobic Surfaces in Aqueous Solutions. Langmuir 2015, 31, 7317-7327.

(54) Bico, J.; Thiele, U.; Quéré, D. Wetting of textured surfaces. Colloids and Surfaces A: Physicochemical and Engineering Aspects 2002, 206, 41-46.

(55) Young, T. III. An essay on the cohesion of fluids. Philosophical Transactions of the Royal Society of London 1805, 95, 65-87.

(56) Tuteja, A.; Choi, W.; Mabry, J. M.; McKinley, G. H.; Cohen, R. E. Robust omniphobic surfaces. Proceedings of the National Academy of Sciences 2008, 105, 18200-18205.

(57) Kim, J. G.; Choi, H. J.; Park, K. C.; Cohen, R. E.; McKinley, G. H.; Barbastathis, G. Multifunctional inverted nanocone arrays for non-wetting, self-cleaning transparent surface with high mechanical robustness. Small 2014, 10, 2487-2494. 
(58) Babakhani, B.; Ivey, D. G. Anodic deposition of manganese oxide electrodes with rodlike structures for application as electrochemical capacitors. Journal of Power Sources 2010, 195, 2110-2117. 


\section{Graphical TOC Entry}

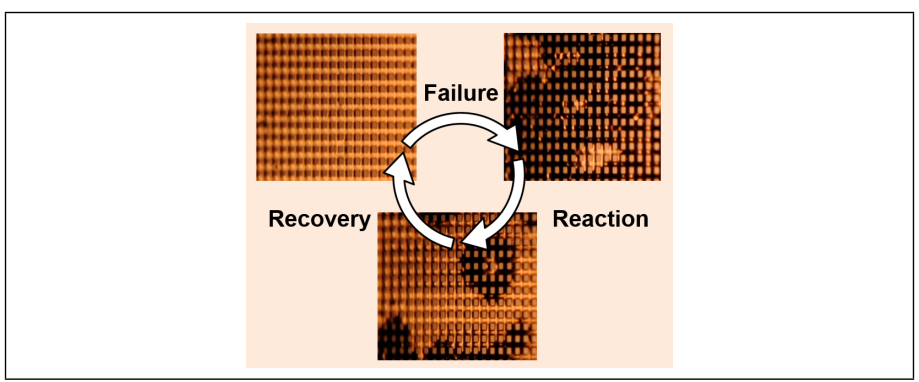

